\title{
INNOVACIÓN Y FORMACIÓN PROFESIONAL: RELACIONES ENTRE SISTEMAS, POLÍTICAS Y ACTORES
}

\author{
INNOVATION AND VOCATIONAL TRAINING: RELATIONS BETWEEN \\ SYSTEMS, POLICIES, AND ACTORS
}

\author{
Ignasi Brunet Icart \\ Juan Rodríguez Soler \\ Universidad Rovira i Virgili, Tarragona. España/Spain \\ ignasi.brunet@urv.cat \\ juan.rodriguez@,urv.cat
}

Recibido/Received: 23/03/2012

Modificado/Modified: 21/09/2012

Aceptado/Accepted: 27/09/2012

\section{RESUMEN}

Este artículo presenta un avance de resultados del proyecto FPInnova, "Formación profesional y sistema de innovación: el papel de los trabajadores intermedios en los procesos de innovación de las pymes industriales" (CSO2011-29410-C03-01), financiado por el Ministerio de Ciencia e Innovación. El objetivo de esta investigación consiste en analizar las relaciones entre el sistema de formación y el sistema regional de innovación de seis regiones. En el contexto actual de crisis económica el estudio de los procesos de construcción institucional y social del modelo económico español se hace más necesario. La educación y la innovación forman parte de la respuesta que debe darse a los retos actuales. Aquí se presenta un primer análisis de estas relaciones para dos de las regiones estudiadas.

\section{PALABRAS CLAVE}

Sistemas regionales de innovación, formación profesional, PYMES.

\section{SUMARIO}

1. Introducción. 2. Elementos teóricos. 3. Estrategia metodológica. 4. Resultados. 5. Conclusiones. Bibliografía.

\begin{abstract}
This article presents some first results from the FPInnova project, "Vocational training and innovation system: the role of intermediate workers in industrial SMEs' innovation processes" (CSO2011-29410C03-01), funded by the Ministry of Science and Innovation. The objective of this research is to analyze the relationship between the training system and the regional innovation system of six regions. In the current context of economic crisis the study of the processes about the institutional and social construction of the Spanish economic model becomes more necessary. Education and innovation are part of the answer to the current challenges. Here is a first analysis of these relationships for two of the regions studied.
\end{abstract}

\section{KEYWORDS}

Regional innovation systems, vocational training, SMEs. 


\section{CONTENTS}

1. Introduction. 2. Theoretical Elements. 3. Methodological Strategy. 4. Results. 5. Conclusions. References.

\section{INTRODUCCIÓN}

Este texto presenta un avance de resultados del proyecto FPInnova, "Formación profesional y sistema de innovación: el papel de los trabajadores intermedios en los procesos de innovación de las pymes industriales" (CSO2011-29410-C03-01), financiado por el Ministerio de Ciencia e Innovación del Gobierno de España. Nuestro objetivo principal es analizar las relaciones entre el sistema de formación profesional (tanto la formación inicial/reglada como la formación para el empleo) y las empresas industriales de sectores de intensidad tecnológica media y alta en España, con especial atención a su influencia sobre los procesos de innovación en las empresas.

Este proyecto de investigación se basa en la perspectiva de los sistemas regionales que incide en la importancia del contexto institucional y social hacia la configuración y desarrollo de un tipo determinado de actividad económica y de procesos de innovación (Lundvall, 1992). La perspectiva de los sistemas regionales de innovación destaca que cada país, cada región (y de hecho cada empresa) construye su propio camino a la innovación. Estudios recientes dentro de esta perspectiva han puesto de manifiesto la especificidad de las capacidades de innovación de las PYME, la importancia de los procesos de aprendizaje interactivo entre empresas y las dificultades en las relaciones con los agentes de I+D. Desde este proyecto se apuesta por incluir el sistema de educación/formación profesional como factor importante a la hora de configurar los procesos de innovación que se desarrollan en las empresas. Nuestra hipótesis de partida apunta a que el sistema de formación profesional resulta un factor importante en la configuración de la actividad económica y la innovación, proceso que, a menudo, se desarrolla a nivel regional.

Actualmente, aunque en España se está desarrollando una reforma nacional de la formación profesional que busca la integración de los diferentes subsistemas que la componen (formación inicial/reglada, formación continua y formación ocupacional), existen limitaciones a nivel de la propia formación profesional (por ejemplo, las relaciones entre los subsistemas de formación profesional y los agentes que los llevan a cabo) y en las relaciones entre el sistema de educación y formación profesional (centros de FP) y las empresas industriales.

Por otro lado, los modelos organizacionales de las empresas industriales españolas y sus estilos de gestión más jerárquicos y menos participativos impiden una contribución real de los trabajadores técnicos intermedios a los procesos de innovación dentro de las empresas, particularmente las PYME industriales, aunque éstas sean de nivel tecnológico medio-alto. Además, el modelo organizacional dominante de baja cualificación, unido a la distancia social y cultural entre ingenieros/científicos y trabajadores técnicos, obstaculiza los procesos de innovación. A partir del proceso de integración de la formación profesional, el acercamiento de ésta (sobre todo en su versión más "alejada" al tejido empresarial como es la formación inicial/reglada) a las empresas (sobre todo, al tejido de PYME industrial) puede contribuir a mejorar los procesos de innovación dentro de éstas. 


\section{PREMISAS TEÓRICAS}

\subsection{Sistemas nacionales y regionales de innovación}

La perspectiva de los sistemas de innovación, que critica la visión ortodoxa del conocimiento como un conocimiento genérico, codificable, accesible sin costo y con independencia del contexto, surge a finales de los años ochenta (Freeman 1987, Dosi et al. 1988) y se desarrolla en la década de los noventa. Bajo esta perspectiva, la innovación es concebida como el resultado de la interacción de actores y factores tanto internos como externos a la empresa en un entorno institucional y cultural (las relaciones y los procesos internos de la empresa, las relaciones con clientes, proveedores y agentes de $\mathrm{I}+\mathrm{D}$, las políticas públicas, el sistema de educación y formación, el sistema de relaciones laborales, el sistema financiero, etc.). En este sentido, el cambio tecnológico es visto como un proceso endógeno condicionado por procesos organizativos, institucionales y culturales. Recientemente, algunos investigadores han distinguido dos "modos de innovación": el modo STI (Science, Technology, Innovation) y el modo DUI (Doing, Using, Interacting) (Jensen et al., 2007; Lundvall y Lorenz, 2007). El primero, típico de los sectores de alta tecnología, se caracteriza por factores como el conocimiento codificado, el personal científico interno y las relaciones con las organizaciones externas S\&T (Science \& Techonology). El segundo modo, en cambio, se caracteriza por factores como el conocimiento tácito, la interacción entre personas y entre departamentos, y una estrecha relación empresas y usuarios y/o clientes.

Dentro de la perspectiva de los sistemas nacionales de innovación, la aplicación a nivel regional ha recibido una atención cada vez mayor (Storper, 1997; Isaksen y Asheim, 2003). Estudios dentro de esta perspectiva (la de los Sistemas Regionales de Innovación, o más conocidos por su acrónimo anglosajón, RIS) han señalado la importancia del nivel regional de los procesos de innovación de las PYME en aspectos como el intercambio de conocimiento tácito y el desarrollo de grupos de trabajadores cualificados (Kauffmann y Tödtling, 2003; Asheim y Coenen, 2005), enmarcándose, por tanto, bajo la perspectiva del modo de innovación DUI. De hecho, bajo nuestro punto de vista, gran parte de la literatura sobre sistemas regionales de innovación resulta compatible con este modelo. Bajo esta perspectiva la innovación es vista como un proceso de aprendizaje interactivo tanto interno (la comunicación y las relaciones entre los niveles jerárquicos y entre los departamentos funcionales) como externo a la empresa (relaciones con clientes, proveedores, agentes de $\mathrm{I}+\mathrm{D}$, etc.). Estos procesos de aprendizaje tanto internos como externos se llevan a cabo dentro de un entorno institucional y cultural determinado (relaciones entre sectores público y privado, sistema de relaciones laborales, entorno productivo, sistema de educación y formación; sistema financiero). El modelo de aprendizaje interactivo destaca la importancia del conocimiento tácito, que se caracteriza por ser específico y dependiente del contexto (en contraste con el conocimiento codificado y, por tanto, fácilmente transmitible del modo STI). La proximidad espacial y social entre los diferentes actores se convierten en catalizadores de fomento de la confianza y la transferencia de conocimiento (Asheim y Gertler, 2005).

Bajo la perspectiva de los sistemas regionales de innovación, los estudios sobre los procesos de innovación del tejido empresarial de PYME se han centrado en aspectos tales la cooperación con clientes y proveedores, y las relaciones con los agentes de I+D (Fritsch, 2001; Asheim y Coenen, 2005). En particular, estos estudios han demostrado la importancia de los procesos de aprendizaje interactivo con unos pocos clientes y 
proveedores, y las dificultades en las relaciones con los agentes de I+D, tales como centros tecnológicos y universidades. Los procesos internos de aprendizaje de las PYME y las relaciones entre estas empresas y los agentes de educación y formación ha recibido, en cambio, mucha menos atención.

\subsection{Educación y sistema de formación e innovación}

La perspectiva de los sistemas de innovación ha ampliado el concepto de innovación a fin de incluir aspectos organizativos, institucionales y culturales. El modelo interactivo y la literatura de los RIS han señalado la importancia de las actividades de producción rutinarias (learning by doing), de la adaptación de tecnologías y sistemas (learning by using) y de la interacción entre los agentes internos y externos a la empresa (clientes, proveedores, agentes de I+D) (“aprendizaje por interacción”). En esta investigación se quiere dar un paso más y explorar las relaciones entre el "sistema de desarrollo de recursos humanos" (especialmente la educación y formación profesional) y el sistema de innovación (con especial atención al papel de los trabajadores intermedios dentro de los procesos de innovación de las empresas).

Algunos autores han llamado la atención sobre la importancia de la relación entre el sistema de educación y formación y el sistema de innovación (entre otros, Lundvall, 2002). Como estos investigadores apuntan, los sistemas de innovación tienen su origen en los sistemas de producción y los sistemas de desarrollo de recursos humanos. Los sistemas de educación y los mercados laborales están nacionalmente constituidos y juegan un papel clave en la creación de competencias y en la conformación de las bases de los procesos de innovación. En los últimos años, algunos estudios internacionales han analizado las relaciones entre las diferencias institucionales, el aprendizaje dentro de la organización y el modo de la innovación (Arundel et al., 2007; Lundvall, Rasmussen y Lorenz, 2008). En este sentido, se ha señalado que el cuello de botella para mejorar la capacidad innovadora de las empresas europeas no se sitúa en los bajos niveles de gastos en $\mathrm{I}+\mathrm{D}$, sino en la presencia generalizada de unos entornos de trabajo que son incapaces de proporcionar un ambiente fértil para la innovación (Arundel et al., ibíd.). Refiriéndose a los países del sur europeo, Lundvall, Rasmussen y Lorenz (2008: 689) sugiere que "un cuello de botella para la construcción de organizaciones de aprendizaje en las economías menos desarrolladas de Europa parece estar en el nivel de formación profesional. Portugal, España, Italia y Grecia, todos los cuales han dado pasos importantes en el aumento del nivel de graduados en ciencias e ingeniería, se destacan por sus bajos niveles de inversión en la formación profesional continua y de menor rango en la escala de aprendizaje discrecional".

Recientemente, algunos autores han señalado la importancia del sistema de formación profesional y continua para la innovación (Rosenfeld, 1998; Bosch y Charest, 2008; Toner, 2009). En su informe para la OCDE, Rosenfeld (1998) sugiere que los institutos de educación técnica y de formación profesional (EFP) estaban en una posición mejor que las universidades para ponerse al servicio de las PYME. En este sentido, Rosenfeld propone una interesante taxonomía de las misiones de los centros de EFP que van desde la educación formal a través de mejorar las competencias y capacitación de los trabajadores hasta ayudar a las PYME en el despliegue de la tecnología, la innovación y la cooperación entre empresas. Este y otros estudios señalan también la importancia de que los institutos de formación profesional cuenten con personal con experiencia en la industria y en el papel que los institutos de formación profesional pueden desempeñar en los sistemas de innovación, tanto locales y regionales. Estas características entroncan con las funciones de 
los centros integrados de formación profesional desarrollados a lo largo del territorio español. Sin embargo, a menudo existen barreras que dificultan la participación de institutos de formación profesional en estas funciones no tradicionales, como la falta de autonomía y flexibilidad de los centros, la falta de incentivos o medidas políticas adecuadas y la falta de relaciones con los agentes de $\mathrm{I}+\mathrm{D}$, como los centros tecnológicos. Por otro lado, para el caso español, existe una falta de coordinación entre el sector de la educación profesional y el sistema de formación de los trabajadores, gestionada de forma mayoritaria a nivel nacional por las organizaciones empresariales y sindicales (Homs, 2008).

Como Bosch y Charest (2008) han señalado, el sistema de educación técnica y formación profesional (EFP) está profundamente arraigado y condicionado por el tejido productivo de cada país, así como los diferentes mercados laborales nacionales, los sistemas de relaciones laborales, etc. Diversos estudios apuntan a las diferencias entre las llamadas economías de mercado "liberales" (entre otros, EE.UU., Reino Unido) y las economías de mercado "coordinadas" (entre otros, Francia, Alemania, etc.), y resaltan el desarrollo y el prestigio de la EFP en las segundas (como se ejemplifica en el "sistema dual" alemán) (Rubery y Grimshaw, 2003; Bosch y Charest, 2008; Toner, 2009). Esta literatura muestra que las economías de mercado coordinadas, que tienden a tener una fuerte base manufacturera, han tenido bastante éxito en la construcción de una base institucional que puede ayudar a resolver el problema de la "caza furtiva" o del free rider (es decir, aquel que corre con los gastos y los beneficios de la formación). Factores tales como la existencia de un mercado laboral ocupacional basado en la cualificación reconocida a nivel nacional, la escasa distancia organizacional entre el personal ingeniero y el personal técnico, una visión a largo plazo de las relaciones laborales, y un sistema de formación profesional sólido, basado en una plataforma de habilidades genéricas y adaptables favorecen la inversión en formación por parte de empleadores y empleados y la introducción y despliegue de nuevas tecnologías. Por el contrario, esta literatura señala que el modelo del Reino Unido combina un modo de innovación S\&T “elitista" en industrias de alta tecnología con otra basada en un bajo nivel de cualificación, limitado y específico de la empresa que se presenta en otros sectores industriales.

Autores como Toner (2009) entre otros afirman que los puestos intermedios técnicos cualificados por el sistema de EFP, hacen (o pueden hacer) una importante contribución a la innovación en las empresas como resultado de su compromiso práctico en el diseño, instalación, operación y mantenimiento de productos y procesos. Los productos y servicios intensivos en innovación requieren una mano de obra cualificada capaz de una rápida adaptación a los procesos de trabajo y la innovación continua de productos. El rol de estos trabajadores sería particularmente importante en el learning-by-doing. La complementariedad entre la educación, la formación y la innovación parece evidente, pero como asegura Toner (Ibíd.: 61), "a pesar de reconocer el papel central de alto nivel y la distribución más amplia de cualificaciones de los trabajadores en la promoción de la innovación, ha habido poca investigación en el campo de los estudios de innovación, o incluso de otras disciplinas, sobre este tema".

Las reformas de la EFP en España que se iniciaron en la década de los noventa todavía están muy lejos de hacer frente a problemas como el bajo nivel de calificaciones en la formación secundaria postobligatoria excluido bachillerato (OCDE, 2009), la conexión insuficiente entre la formación profesional y las necesidades del tejido empresarial industrial (CES, 2009), la falta de un sistema nacional de cualificaciones y el 
reconocimiento de las competencias, la falta de coordinación entre el sistema de formación profesional y el sistema de formación de los trabajadores (Homs, 2008; CES, ibíd.), y los problemas de gobernabilidad entre las autoridades nacionales y regionales (Homs, ibíd.). Por otro lado, el problema de la "caza furtiva" está muy presente en España (los empleadores parecen tener miedo de que unos trabajadores mejor formados podrían perjudicar a la empresa o reclamar salarios más elevados) (Berechet, Huerta y San Miguel, 2008), y los grandes sectores de la industria española parecen estar dominados por un modelo jerárquico de la organización con una baja cualificación y contribución de los trabajadores en la empresa (Huerta, 2003). Estudios recientes (Olazarán, Albizu y Otero, 2008; Lavia et al., 2010) muestran que las PYMES industriales de los sectores de tecnología media resultan bastante innovadoras, pero sus procesos de innovación todavía poseen obstáculos importantes a la hora de incluir a sus trabajadores técnicos en dichos procesos de innovación. Estos obstáculos responden a un modelo organizacional de baja cualificación, jerárquico y poco participativo, aspectos que limitan los procesos de aprendizaje interactivo internos y las relaciones con los centros de FP, como proveedores de recursos humanos pero también como posibles proveedores de servicios y/o acciones de mejora de productos, procesos, organización, etc. Esta investigación pretende incidir en estos aspectos, focalizando la atención en aquellos casos en donde la interacción entre el sistema de formación profesional y el tejido empresarial industrial va más allá de estas limitaciones aparentemente características del caso español.

\section{ESTRATEGIA METODOLÓGICA}

\subsection{Aspectos generales de la investigación}

La investigación que aquí se presenta consiste en un estudio comparativo de las relaciones entre el sistema de educación y formación profesional (EFP) y los procesos de innovación en pymes industriales en seis Comunidades Autónomas españolas: Aragón, Asturias, Cataluña, Madrid, Navarra y País Vasco. La metodología a desarrollar combina las estrategias cuantitativa y cualitativa, bajo el formato de estudios de casos para cada una de las regiones a investigar. Los estudios de casos regionales se están llevando a cabo por investigadores de universidades de las seis Comunidades Autónomas que participan: Universidad de Oviedo (Asturias), Universidad de Zaragoza (Aragón), Universidad Rovira i Virgili (Cataluña), la Fundación Ideas (Comunidad de Madrid), Universidad Pública de Navarra (Región de Navarra) y Universidad del País Vasco (País Vasco).

\subsection{Metodología y plan de trabajo}

La investigación consta de tres grandes fases, diferenciadas desde el punto de vista secuencial/temporal, metodológico y respecto a los objetivos a conseguir en cada una de estas fases. A continuación se describen de forma sucinta, atendiendo a sus objetivos y a las técnicas utilizadas.

\section{Fase 1: Investigación exploratoria}

Esta fase se centra en el análisis del sistema de innovación regional con especial atención a la función del sistema de formación profesional y para el empleo. El objetivo de esta fase es relacionar el sistema de formación profesional con el sistema regional de innovación y establecer el papel desempeñado por los agentes del sistema de educación formal y para el 
empleo en cada uno de los territorios analizados. Para ello se han realizado entrevistas exploratorias a agentes clave de los sistemas de innovación, formación profesional y de formación para el empleo: administración regional (áreas de empleo y educación), agentes sociales, centros de formación profesional, análisis documental de políticas, planes, programas y/o iniciativas relacionadas con los sistemas de innovación, formación profesional y para el empleo en cada región, y análisis de bases de datos estadísticos.

\section{Fase 2: Investigación cualitativa}

El objetivo de la fase 2 es llevar a cabo un estudio prospectivo sobre cómo los centros de formación profesional contribuyen al desarrollo del capital humano que promueve los procesos de innovación en las pymes industriales. Para ello, se realizarán una serie de entrevistas en profundidad a diferentes perfiles en función de si hablamos de centros de formación o de empresas. Para el primer caso, se realizarán entrevistas a responsables del centro con vinculación con las empresas con los siguientes perfiles: Director, Tutores de FCT, Responsable de relaciones con empresas y Egresados. Para el caso de las empresas, se realizarán entrevistas a los siguientes perfiles disponibles: Director, Responsable recursos humanos, Responsable de I+D, Trabajadores técnicos. El número de entrevistas variará en función del tipo de centro y/o empresa.

\section{Fase 3: Investigación cuantitativa}

El propósito de la fase 3 es llevar a cabo una encuesta a pymes industriales en las seis regiones, a fin de evaluar la importancia que los centros de formación profesional y, en consecuencia, el capital humano desarrollado en ellos, tienen en la actividad innovadora de dichas empresas. Para ello se realizará una encuesta telefónica a una muestra de pymes industriales en sectores de intensidad tecnológica media-alta.

\section{PRIMEROS RESULTADOS}

A continuación se presenta un avance de resultados correspondientes a la finalización de la primera fase, a partir del análisis exploratorio de cada uno de los territorios seleccionados. Aquí se apuntan sólo las conclusiones de los casos correspondientes a Cataluña y Aragón.

\subsection{Caracterización de los territorios: políticas y actores clave Aragón}

Del análisis realizado se deriva que la Comunidad de Aragón todavía tiene margen de desarrollo respecto de su sistema de innovación. Debido a las características del propio territorio, la mayoría de agentes se concentran en la conurbación de Zaragoza. El resto de territorio se considera medio rural, según algunos entrevistados, habiendo iniciativas puntuales vinculadas con actividades productivas muy concretas. La tipología de empresas pequeñas genera una serie de obstáculos. Aunque el entorno facilita un mayor conocimiento e interacción por parte de los diferentes agentes.

Según el Gobierno de Aragón, los agentes de innovación en el territorio se distribuyen entre las 2 universidades existentes, 3 centros de innovación y tecnología, 4 institutos universitarios de investigación, 6 centros del CSIC, 6 oficinas de transferencia de resultados de investigación y 3 parques tecnológicos, algunos de reciente creación. 
De las entrevistas y documentación analizadas se deduce que la apuesta de la Administración Regional por la potenciación y el desarrollo de la innovación y la investigación en la Comunidad de Aragón es relativamente limitada en el tiempo, lo que permite en la actualidad un importante margen de actuación. La mayoría de iniciativas, planes y/o programas vinculadas a este ámbito son de reciente creación y tienen todavía un escaso recorrido (en algunos casos, entre 5 y 10 años).

La política aragonesa de innovación y desarrollo se gestiona desde el Departamento de Industria e Innovación del Gobierno de Aragón. Existen dos planes regionales de impulso de la innovación y la investigación. El I Plan Autonómico de Investigación, Desarrollo y Transferencia de Conocimientos de Aragón (PAID) abarca el período entre 2002 y 2004, dentro del cual se aprobó la Ley 9/2003 de fomento y coordinación de la investigación, el desarrollo y la transferencia de conocimientos en Aragón ("Ley de la Ciencia"). El II PAID mantiene las mismas líneas de actuación y comprende el período de 2005 y 2008 . Estas iniciativas no parecen haber tenido continuidad en el tiempo. Durante la vigencia de este II PAID se crean diferentes iniciativas vinculadas con la difusión y promoción de la investigación.

La política de la Administración aragonesa en materia de formación profesional se realiza desde el Departamento de Educación, Universidad, Cultura y Deporte junto con el Departamento de Hacienda, Economía y Empleo. La relación entre estos dos departamentos es, según la mayoría de los entrevistados, muy fluida, sobre todo si es comparada con otras Comunidades Autónomas. Aún así, se observan algunas discrepancias entre estos dos departamentos que atienden, en algunos casos, a personalismos. En este sentido, la iniciativa de los Centros Integrados ha supuesto un mayor acercamiento entre ambos departamentos. La relación entre ambos departamentos se incrementa a partir de las transferencias en educación, en 1998, y en empleo, en 2000. Desde entonces la colaboración parece ser continuada y permanente.

La formación profesional en Aragón se concreta en diferentes políticas, planes y programas. Entre ellos destaca el Plan de Formación e Inserción Profesional de Aragón y Acuerdo Económico y Social para el Progreso de Aragón (AESPA) como marco general, los dos Planes Aragoneses de Formación Profesional y, en menor medida, los dos Planes Generales de Educación Permanente de Aragón. Además de los dos planes de formación profesional, se destacan diferentes órganos institucionales tales como el Instituto Aragonés de Empleo (INAEM), el Consejo Aragonés de Formación Profesional (CAFP) y, en menor medida el Consejo de Educación Permanente de Aragón (CEPA).

La iniciativa más importante es la apuesta por los centros integrados de formación profesional. En Aragón, se inició en 2005 una fase experimental, creando 5 centros integrados experimentales. Estos 5 centros experimentales, en septiembre de 2010, se constituyen como Centros Públicos Integrados de Formación Profesional.

\section{Cataluña}

De las entrevistas realizadas y la documentación analizada se deriva un vasto y complejo sistema de innovación, condicionado por un tejido productivo más o menos desarrollado en el territorio y por una serie de actores e instituciones vinculadas a la investigación y a la innovación. Cabe destacar que predominan los agentes públicos especialmente vinculados con la investigación (entre otras, las universidades catalanas y sus derivados), aunque la transferencia de tecnología tiene un cierto protagonismo (centros tecnológicos, red TECNIO, entre otros). El sistema catalán de investigación e innovación 
está integrado fundamentalmente por las 12 universidades, 22 centros tecnológicos, 77 centros de investigación (incluyendo centros CSIC, centros TECNIO, etc.), 9 instituciones hospitalarias, las grandes infraestructuras de soporte a la investigación, 23 parques científicos y tecnológicos, y las redes y grupos de investigación.

La apuesta del Gobierno catalán por el desarrollo y fomento de la innovación se manifiesta en la sucesión de planes y acuerdos generales relacionados con ello. Entre ellos, destacan el Pacto Nacional para la Investigación y la Innovación como marco general y que supone la hoja de ruta en materia de investigación e innovación para los próximos 15 años. Impulsado por el Departamento de Innovación, Universidades y Empresa, este pacto ha sido firmado por el Gobierno catalán, los partidos políticos con representación parlamentaria, los principales agentes sociales y todas las universidades catalanas.

Entre los planes que marcan las directrices de la política catalana de investigación e innovación destacan, además, los sucesivos Planes de Investigación e Innovación (PRI) (2005-2008 y 2010-2013). Previamente, entre 1993 y 2004 en Cataluña se aprueban tres sucesivos planes de investigación. Tras el tercer plan se da una convergencia entre el fomento del sistema de investigación y el fomento del sistema de innovación, dando lugar a los dos PRI.

Desde el punto de vista de las políticas de formación profesional, en Cataluña existen dos tipos de acuerdos o planes generales vinculados al impulso de la formación profesional en el territorio catalán: los dos planes de formación profesional y el Acuerdo Estratégico para la internacionalización, la calidad del empleo y la competitividad de la economía catalán con su posterior revisión. Habría que añadir los diferentes planes de ocupación (que desembocan en la Estrategia Catalana de Ocupación en 2006) y el reciente Plan de Desarrollo de las políticas activas de empleo de Cataluña (2010-2011).

Sin embargo, estas últimas políticas no inciden tanto en el desarrollo de la formación profesional como el Acuerdo estratégico, donde se establece como prioridad el desarrollo y consolidación de un sistema integrado de formación profesional adaptado al tejido productivo del territorio catalán. Esta directriz es una de las pocas que se mantienen inalterables en su posterior revisión en 2008. El acuerdo estratégico sigue los pasos del II Plan Catalán de Formación Profesional, donde se establecen las bases para crear un sistema integrado de formación profesional, incidiendo en dos elementos: la consolidación del sistema integrado de formación y la adaptación de ésta a las necesidades de la economía catalana.

Más allá de estos planes generales, a partir de las entrevistas y la documentación analizada destacan diferentes órganos institucionales con incidencia en la formación profesional de Cataluña. Algunos de estos órganos son el Consell Català de Formació Profesional (CCFP), el Servei d'Ocupació de Catalunya (SOC), y, en menor medida, el Consell Superior d'Avaluació del Sistema Educatiu (CSASE).

En cuanto a los programas o iniciativas vinculadas a la formación profesional, destaca por encima del resto, la red de centros FP.CAT, que la integran los Centros de Innovación y Formación Ocupacional (CIFO), del SOC, y algunos IES, teniendo en cuenta su experiencia en oferta integral de formación profesional. El objetivo de este programa es crear una red de centros que ofrezcan formación profesional de los diferentes subsistemas de formación.

\subsection{Relaciones entre sistemas: innovación y formación profesional Aragón}

Podemos definir a la Comunidad de Aragón como una región próspera, atendiendo a sus niveles de PIB, renta per cápita, VAB de su industria, empleo, etc., sobre todo en 
relación al conjunto del Estado (Todos los datos estadísticos utilizados en la investigación están extraídos de las siguientes fuentes: Instituto Nacional de Estadística (INE), Eurostat, Fundación Tripartita para la Formación en el Empleo y el anuario estadístico Las cifras de la Educación en España (Ministerio de Educación, Cultura y Deporte). Esto se debe, en parte, a un no desdeñable tejido industrial, con un elevado nivel tecnológico. De hecho, de las seis regiones estudiadas Aragón es la segunda región con mayor nivel tecnológico dentro del sector industrial. Por otro lado, el nivel de cualificación general de su población resulta también elevado, relativamente superior a la media española. Cabe destacar también el importante peso de la formación profesional entre la población ocupada, especialmente los Grados Medios si hablamos del sector industrial.

Sin embargo, en términos de $\mathrm{I}+\mathrm{D}$, la Comunidad de Aragón no destaca por su esfuerzo inversor, ello a pesar del mayor protagonismo que tiene la Administración Regional en la ejecución de dicho gasto, por encima de la media estatal. De hecho, son las empresas aragonesas las que ejecutan (en mayor medida que la media española) las acciones innovadoras dentro del territorio. Esta menor inversión general se nota en el menor volumen de personal dedicado a $\mathrm{I}+\mathrm{D}$ sobre la población ocupada. De este personal, el sector de la enseñanza superior es el que aglutina un mayor volumen. Aunque esto ha permitido un crecimiento mayor del personal dedicado a I+D sobre el conjunto del Estado.

Respecto al gasto en I+D cabe destacar el protagonismo de las empresas industriales, muy superior a la media española. Aunque este gasto responde en menor medida al desarrollo interno de $\mathrm{I}+\mathrm{D}$ o a la adquisición externa de $\mathrm{I}+\mathrm{D}$, y más a la adquisición de conocimientos externos de forma, por cierto, muy superior al resto de regiones estudiadas.

El acceso a ayudas a la $\mathrm{I}+\mathrm{D}$ resulta capital para potenciar la innovación entre las empresas aragonesas. Aunque, si bien el acceso a ayudas provenientes de la Administración Central es superior que la media española, el acceso a ayudas regionales y provenientes de la Unión Europea es muy inferior. Esto quizás sea una de las razones por las que las empresas aragonesas cooperan en mayor medida que las del conjunto del Estado. Otra de las posibles razones sea el limitado territorio en el que las empresas aragonesas se ubican, así como la tipología de éstas.

El complejo de centros de formación e investigación parece estar dando una relativa cobertura a las necesidades, tanto de capital humano como de innovación e investigación. Aún así, muchos de los agentes vinculados con el fomento y desarrollo de la innovación son de reciente creación. Respecto de la formación, el fomento de la relación entre formación profesional inicial/reglada y la formación para el empleo (sea ésta de oferta o demanda) tiene un bagaje limitado en el tiempo. Esta relación se materializa (desde el punto de vista de los centros públicos) en la experiencia de los Centros Integrados de Formación Profesional (CPIFP), de reciente creación, partiendo de una experiencia piloto desde 2005. Hasta la fecha se observa un relativo apoyo por parte del INAEM a estos centros a partir de la partida propia de formación de oferta dirigida prioritariamente a desempleados. Sin embargo, no parece desprenderse avances en la participación de estos CPIFP en la formación de oferta dirigida a ocupados y/o otros programas de formación (por parte del INAEM en calidad de asignación presupuestaria propia o simplemente de participación en la asignación de los cursos de esta partida). En este sentido, la colaboración con los agentes sociales para coparticipar con los CPIFP (ofreciendo aulas y docentes) está en una situación prematura y poco consolidada. Actualmente se están realizando contactos de forma puntual e individualizada. 
Respecto a la relación entre centros educativos y empresas, la relación que inician, desarrollan y consolidan los CPIFP con el tejido empresarial tiene tres consecuencias. La primera es una mejora en la inserción de los egresados de estos centros. La segunda es un acercamiento de las empresas a la formación reglada (tradicionalmente distanciados), lo que, a su vez, provoca un aumento exponencial de los contactos entre ambos actores (vía jornadas, formación a demanda, prestación de servicios, estancias de profesorado, etc.). La tercera consecuencia es que se da una mejor detección de necesidades, pudiéndose convertir los CPIFP en "informantes clave" para la mejor detección de estas necesidades (formativas, de mejora de sistemas de producción, implementación de sistemas de innovación,...), más allá de observatorios y jornadas técnicas planteadas y organizadas desde la Administración Regional. Ello permite crear relaciones más allá de la dación de formación por parte de los centros de formación profesional, generándose acciones vinculadas con la mejora de productos y/o servicios a las empresas o la difusión del conocimiento y la innovación.

\section{Cataluña}

Cataluña se caracteriza por ser una región con una capacidad de desarrollo importante, debido, en parte a unos elevados niveles de PIB, una relativa renta per cápita (es la cuarta región de las seis estudiadas, pero supera la media nacional), un VAB industrial importante, así como los niveles de empleo dentro del sector industrial, siempre respecto a la media española. Esto es debido a la existencia de un importante tejido industrial, extendido por todo el territorio catalán, con unos elevados niveles tecnológicos (en comparación con el conjunto del Estado). Si lo comparamos con el resto de regiones estudiadas, Cataluña ocuparía una posición intermedia. En este sentido, el nivel de cualificación general de su población ocupa también una posición media, con proporciones similares a la media del conjunto del Estado. El peso de los estudios profesionales en el sector industrial es limitado. De hecho, Cataluña es, después de Madrid, la región cuya población ocupada en la industria con estudios profesionales ha crecido menos en los últimos 25 años. Esta situación apunta a un uso menor que en otras regiones industrializadas del personal con cualificaciones profesionales de grado medio-superior, lo que puede indicar un débil ajuste entre las necesidades del tejido industrial catalán y las formaciones profesionales intermedias.

En cuanto al gasto en $\mathrm{I}+\mathrm{D}$, Cataluña supera a la media estatal, pero ocupa una posición intermedia entre las seis regiones estudiadas. En este sentido, son las empresas catalanas las que ejecutan (en mayor medida que la media española) las acciones innovadoras dentro del territorio. Esta menor inversión general se nota en el menor volumen de personal dedicado a I+D sobre la población ocupada. De este personal, cabe destacar, además del sector empresarial, del significativo peso de la Administración pública.

En cambio, si nos limitamos al sector industrial, respecto al gasto en I+D destaca el protagonismo de las empresas industriales, muy superior a la media española y al resto de regiones estudiadas. Este gasto responde, sobre todo, a la adquisición externa de I+D y no tanto a al desarrollo interno de I+D.

El acceso a ayudas a la $\mathrm{I}+\mathrm{D}$ resulta fundamental para entender la capacidad de innovación de las empresas catalanas. Aunque, si bien el acceso a ayudas provenientes de la Administración Central es superior que la media española y al resto de regiones, con el acceso a ayudas regionales y provenientes de la Unión Europea ocurre la situación contraria: es el menor de las seis regiones estudiadas. 
En cuanto al nivel de cooperación de las empresas catalanas, cabe decir que, en términos generales, el nivel de cooperación es limitado, destacando especialmente la cooperación con empresas del mismo grupo, proveedores, clientes y, especialmente, con universidades $\mathrm{y}$, en menor medida, centros tecnológicos.

Esta última característica enlaza con la política de $\mathrm{I}+\mathrm{D}+\mathrm{i}$ catalana que posee cierto recorrido en el tiempo. Aunque cabe destacar que hasta recientemente dicha política se ha centrado más en desarrollar y articular las estructuras del sistema de investigación de Cataluña. La transferencia de tecnología y la cooperación entre agentes del sistema de formación-investigación y el tejido empresarial, sobre todo, el de pymes, resultan más recientes.

En cuanto a la política de formación profesional impulsada en Cataluña cabe destacar el protagonismo de la Administración regional pero, sobre todo, el de los agentes sociales, sobre todo en el tema de la integración de la formación profesional y sus subsistemas. Dicha integración, aunque en la práctica se ha dado en mayor o menor medida en el territorio, desde el punto de vista normativo, existe de forma experimental. En los últimos años se han dado grandes avances en el desarrollo de un modelo catalán de integración de los subsistemas de formación profesional.

Más allá de las políticas, acuerdos y/o programas, Cataluña se caracteriza por tres elementos importantes. El primero es la importancia de la articulación del territorio. El segundo es el elevado recorrido que tienen las empresas y los centros formativos (en cuanto a prácticas en centros de trabajo y otros). El tercero se refiere al protagonismo que actualmente tienen los CIFO respecto a la política de integración de los diferentes subsistemas de formación profesional

Para el primer elemento, del análisis realizado se deriva la importancia que, desde la Administración Regional (tanto Educación como Empleo), se otorga a los diferentes niveles territoriales (provincial, comarcal, local, mancomunado).

La articulación de los diferentes programas de formación para el empleo o experiencias como el plan FP.CAT se corresponden con un intento de articulación (organización y relación entre actores clave) del territorio catalán importante. De hecho, es esta mayor o menor articulación la que marca el nivel de importancia de las experiencias en centros integrales de formación profesional, donde los actores que participan (administración local, agentes sociales territoriales, empresarios) tienen una relación entre ellos consolidada en el tiempo. Esta situación hace que este tipo de experiencias creadas escapen a la lógica más formal de los centros integrados o, si se quiere, de la formación inicial/reglada.

Otro elemento significativo es el hecho de que las experiencias en integración de la formación profesional y para el empleo para el caso de Cataluña no están relacionadas exclusivamente con los tradicionales centros de formación profesional. En Catalunya, paralelamente, se ha apostado por el fomento y desarrollo de la red de Centros de Innovación y Formación Ocupacional (CIFO) (creados en 1995), que integran gran parte de la formación para el empleo y parte de la formación profesional vinculada a los certificados de profesionalidad. En este sentido, el plan FP.CAT se desarrolla en IES pero fundamentalmente en centros CIFO. Este tipo de centros aglutina parte de esta formación, estableciendo vínculos con el tejido empresarial del territorio, aunque la tendencia de este tipo de centros (hasta ahora al menos) era desarrollar en mayor medida la parte de la formación para el empleo que la formación profesional inicial/reglada. 


\section{CONCLUSIONES}

Este texto ha mostrado las bondades de un proyecto de investigación que busca estudiar, por un lado, las relaciones entre los diferentes subsistemas de formación profesional y, por otro lado, las relaciones entre el sistema de formación profesional y los procesos de innovación en pymes industriales. Una de las premisas de partida de esta investigación consiste en reconocer la importancia del sistema regional de innovación en la configuración de estas relaciones. Desde la perspectiva de los sistemas regionales de innovación, el contexto institucional y social determina, en gran medida, el conjunto de relaciones entre actores e instituciones, los cuales configuran dicho sistema.

En este sentido, el estudio de la configuración de los sistemas de innovación y de formación profesional y las relaciones entre ellos muestra la importancia del territorio, en tanto que aglutinador de relaciones y actores característicos de cada uno de los casos estudiados. La perspectiva de los sistemas regionales innovación permite analizar dichas especificidades y, por tanto, sus diferentes situaciones y relaciones: el nivel de desarrollo de políticas e iniciativas de $\mathrm{I}+\mathrm{D}+\mathrm{i}$ y de formación profesional, el tejido empresarial característico del territorio y el nivel de consolidación e interacción de los diferentes agentes institucionales implicados condicionan en gran medida la articulación de los sistemas de innovación y formación profesional de los casos estudiados.

\section{BIBLIOGRAFÍA}

ARUNDEL, A.; LORENZ, E.; LUNDVALL, B.A. y VALEYRE, A. (2007), "How Europe"s economies learn: A comparison of work organization and innovation mode for the EU-15", en Industrial and Corporate Change, vol.16, $\mathrm{n}^{\circ} 6$, pp.1175-1210.

ASHEIM, B.T. y COENEN, L. (2005), "Knowledge bases and regional innovation systems: comparing nordic clusters", en Research Policy, vol. 34, n 8, pp.173-190.

ASHEIM, B. T. y GERTLER, M. (2005), "The Geography of Innovation: Regional Innovation Systems", en J. Fageberg, D. Mowery y R. Nelson (eds.). The Oxford Handbook of Innovation, Oxford, Oxford University Press, pp.291-317.

BERECHET, C.; HUERTA, E. y SAN MIGUEL, F. (2008), Invertir en las personas: Formación y Productividad en Navarra, Navarra: Centro para la Competitividad en Navarra, Institución Futuro.

BOSCH, G. y CHAREST, J. (2008), "Vocational training and the labour market in liberal and coordinated economies", en Industrial Relations Journal, vol. 39, n5, pp.428-447.

CES (2009), Sistema Educativo y Capital Humano, Madrid, CES.

DOSI, G.; FREEMAN, C.; NELSON, R.R.; SILVERBERG, G. y SOETE, L. (eds.) (1988), Technological Change and Economic Theory. London: Pinter.

FREEMAN, C. (1987), Technology, Policy and Economic Performance: Lessons from Japan. London: Pinter.

FRITSCH, M. (2001), “Co-operation in regional innovation systems”, en Regional Studies, vol. 35, nº4, pp.297-307.

HOMS, O. (2008), La Formación Profesional en España: Hacia la Sociedad del Conocimiento. Barcelona: Fundación "La Caixa".

HUERTA, E. (ed.) (2003), Los desafíos de la competitividad: La Innovación organizativa y tecnológica en la empresa española. Bilbao: Fundación BBVA.

ISAKSEN, B.T. y ASHEIM, A. (2003), "SMEs and the regional dimension of innovation", en B.T. Asheim, A. Isaksen, C. Nauwelaers y F. Tödtling (eds.). Regional innovation policy and small-medium enterprises. Cheltenham: Edwar Elgar, pp. 21-46. 
JENSEN, M.B.; JOHNSON, B.; LORENZ, E. y LUNDVALL, B.A. (2007), "Forms of knowledge and modes of innovation", en Research Policy, vol. 36, n5, pp. 680-693.

LAVIA, C., OTERO, B.; OLAZARÁN, M. y ALBIZU, E. (2011), "Innovación y territorio: Una encuesta a pymes industriales", en Revista Internacional de Sociología, vol. 69, n², pp. 461-486.

LUNDVALL, B.A. (1992), National Systems of Innovation: Towards a Theory of Innovation and Interactive Learning. London: Pinter.

LUNDVALL, B.A. (2002), Innovation, Growth and Social Cohesion: the Danish Model. Cheltenham: Edward Elgar.

LUNDVALL, B.A. y LORENZ, E. (2007), Modes of Innovation and Knowledge. Taxonomies in the Learning economy. Oslo: CAS workshop on Innotavion in Firms.

LUNDVALL, B.A.; RASMUSSEN, P. y LORENZ, E. (2008), "Education in the Learning Economy:

A European Perspective", en Policy Futures in Education, vol. 6, n6, pp. 681-700.

OCDE (2009), Education at a Glance. Paris: OCDE.

OLAZARÁN, M.; ALBIZU, E. y OTERO, B. (2008), Innovación en las pequeñas y medianas empresas industriales guipuzcoanas. Bilbao: UPV-EHU, Servicio de Publicaciones.

ROSENFELD, S. (1998), Technical colleges, technology deployment and regional development. Modena: OCDE.

RUBERY, J. y GRIMSHAW, D. (2003), The Organization of Employment. An International Perspective. Basingstoke: Palgrave Macmillan.

STORPER, M. (1997), The Regional Economy. NewYork: Guilford Press.

TONER, P. (2009), Workforce Skills and Innovation: An Overview of Major Themes in the Literature. Paris: OCDE.

\section{Breve currículo:}

\section{Ignasi Brunet Icart}

Catedrático de Sociología por la Universidad Rovira i Virgili y director del Grupo de Investigación Análisis Social y Organizativa. Sus líneas de investigación se centran en la sociología de las organizaciones, el desarrollo y el trabajo, la sociología económica, el género y la sociología de la educación.

\section{Juan Rodríguez Soler}

Profesor asociado por la Universidad Rovira i Virgili y miembro del Grupo de Investigación Análisis Social y Organizativa. Sus líneas de investigación se centran en las relaciones laborales, las políticas de empleo, la sociología del desarrollo y el trabajo. 